\title{
Comparison of acquisition and iterative reconstruction parameters in abdominal computed tomography-guided procedures: a phantom study
}

\author{
Julien Frandon ${ }^{1}$, Philippe Akessoul ${ }^{1}$, Aymeric Hamard ${ }^{1}$, Edinaud Bezandry ${ }^{1}$, Romaric Loffroy ${ }^{2}$, \\ Takieddine Addala ${ }^{1}$, Martin M. Bertrand ${ }^{3}$, Jean-Paul Beregi ${ }^{1}$, Joël Greffier ${ }^{1}$ \\ ${ }^{1}$ Department of Medical Imaging, Nîmes University Hospital, University of Montpellier, Medical Imaging Group Nîmes, EA 2992, Nîmes, France; \\ ${ }^{2}$ Department of Vascular and Interventional Radiology, Image-Guided Therapy Center, ImViA Laboratory-EA 7535, François-Mitterrand University \\ Hospital, Dijon, France; ${ }^{3}$ Digestive Surgery Department, Nîmes University Hospital, Nîmes, France
}

Contributions: (I) Conception and design: J Greffier, J Frandon, JP Beregi; (II) Administrative support: JP Beregi, R Loffroy; (III) Provision of study materials or patients: J Greffier, J Frandon, MM Bertrand; (IV) Collection and assembly of data: J Frandon, P Akessoul, J Greffier, A Hamard, T Addala, E Bezandry, MM Bertrand; (V) Data analysis and interpretation: J Frandon, P Akessoul, J Greffier, A Hamard, JP Beregi, MM Bertrand, R Loffroy; (VI) Manuscript drafting: All authors; (VII) Final approval of the manuscript: All authors.

Correspondence to: Julien Frandon, MD, PhD. Département d'Imagerie Médicale, CHU Nîmes, 4 rue du Professeur Robert Debré, 30029 Nîmes, France. Email: julien.frandon@chu-nimes.fr.

Background: Many computed tomography (CT) navigation systems have been developed to help radiologists improve the accuracy and safety of the procedure. We evaluated the accuracy of one CT computer-assisted guided procedure with different reduction dose protocols.

Methods: A total of 128 punctures were randomly made by two operators on two different anthropomorphic phantoms. The tube voltage was fixed to $100 \mathrm{kVp}$. Tube currents (mAs) were defined to obtain 4 dose levels: $180 \mathrm{mAs}$ (D1.00), $90 \mathrm{mAs}$ (D0.50), $45 \mathrm{mAs}$ (D0.25) and $15 \mathrm{mAs}$ (D0.10) with respective volume CT dose index (CTDIvol) of 7.02, 3.52, 1.75 and $0.59 \mathrm{mGy}$. The raw data were reconstructed using level 2 of advanced model-based iterative reconstruction (ADMIRE) (A2) for D1.00, A3 for D0.50, A4 for D0.25 and A5 for D0.10. Two 12-mm targets per phantom were selected. The mean Euclidean distance (EuD) between the tip of the needle and the isocenter of the target was measured for each puncture. The different measures were compared by paired Student's $t$-tests.

Results: The mean EuD was $7.0 \pm 3.1 \mathrm{~mm}$ for the 128 punctures performed. Regardless of which phantom was considered, no significant difference in accuracy occurred between the 4 dose levels, which were $7.1 \pm 3.5 \mathrm{~mm}$ for D1.00; 7.1 $\pm 3.1 \mathrm{~mm}$ for D0.50; 7.2 $\pm 3.0 \mathrm{~mm}$ for D0.25 and $6.6 \pm 2.6 \mathrm{~mm}$ for D0.10.

Conclusions: Abdominal CT-guided procedures, using computer-assisted navigation and iterative reconstruction algorithms, allow precise punctures on anthropomorphic phantoms with a dose reduction of $-92 \%$ compared to a standard protocol.

Keywords: Imaging guided biopsy; computer-assisted; image reconstruction; low-dose computed tomography (low-dose CT); noise reduction

Submitted Mar 24, 2021. Accepted for publication Jun 21, 2021.

doi: 10.21037 /qims-21-328

View this article at: https://dx.doi.org/10.21037/qims-21-328 


\section{Introduction}

Interventional radiology procedures in oncology that allow physicians to diagnose through percutaneous biopsies or treat various tumors by percutaneous thermoablation have become common minimally invasive practices. Different modalities, including ultrasound, X-ray fluoroscopy, magnetic resonance imaging (MRI) or computed tomography (CT), can be used to help the physician identify the best needle trajectory and guide the needle to the target. Many interventional procedures are now performed under CT guidance because of its availability and its high spatial and contrast resolutions, notably for deep lesions.

Clinical targets are sometimes difficult to reach because they require performing an out-of-plane trajectory to avoid nearby organs. Repetitive helical acquisitions remain mandatory to identify the anatomically safest path because they allow a retrospective analysis of the $3 \mathrm{D}$ volume that can identify vascular structures and organs to avoid (1). Considering the recent awareness of the cancer-inducing risks of radiation, dose optimization is currently an important issue (2-10). Regarding helical acquisitions, international practice guidelines for optimization recommend limiting their number and length $(1,11-13)$ and preferring sequential or fluoroscopic acquisitions when possible $(3,7,10)$.

For complex procedures that require repetitive helicoidal acquisitions, which result in higher radiation doses delivered to the patients $(13,14)$, many CT navigation systems have been developed to help radiologists improve the accuracy and safety of the procedure $(15,16)$. Among them is the Imactis ${ }^{\circledR}$ system, a computer-assisted navigation system that provides virtual electromagnetic guidance of the needle in a $3 \mathrm{D}$ space. Imactis ${ }^{\circledR}$ has been shown to improve accuracy for both simple and complex trajectories and to reduce the number of helical acquisitions required $(17,18)$.

However, dose optimization may not depend only on clinical practice optimization. Recent CT scans with new tools, such as iterative reconstruction algorithms, allow significant dose reductions for helical acquisitions as well $(19,20)$ while maintaining an image quality sufficient for diagnosis or lesion detection (21-23). Optimization in CTguidance protocols for interventional radiology procedures consists of striking an appropriate balance between the radiation dose delivered to the patient and the image quality required to guide the needle. We conjecture that the image quality obtainable under very low dose protocol, which is sufficient for certain diagnosis and detection of lesions, may also be sufficient to guide difficult CT interventions.
The primary objective in the present study was to evaluate the feasibility and accuracy of computer-assisted CT-guided puncture at different reduced doses on two anthropomorphic phantoms. The secondary objectives were to evaluate the impacts of operator experience (junior or senior operator) using two different phantoms with different relative target densities (hyper- or hypodense targets), the obliquity of the trajectory (simple or double obliquity) and the depth of the target (superficial or deep target) on the accuracy of CT-guided procedures at reduced doses.

\section{Methods}

\section{Phantoms}

Two anthropomorphic phantoms (CIRS, Norfolk, VA, USA) were used to perform abdominal punctures. The imageguided abdominal biopsy phantom model 071B (phantom 1) corresponds to a simplified abdominal phantom with 12 hyperdense targets (80 UH for a background of $35 \mathrm{UH}$; diameter from 5 to $12 \mathrm{~mm}$ ) that could mimic retroperitoneal nodes, for example. The triple-modality 3D abdominal phantom model 057A (phantom 2) corresponds to a small adult abdomen with 8 hypodense targets $(30 \mathrm{UH}$ for a background of $75 \mathrm{UH}$; diameter from 5 to $12 \mathrm{~mm}$ ) that can, for example, mimic metastatic liver lesions (Figure 1).

\section{CT protocol}

Acquisitions were performed on a Somatom Definition AS + CT unit (Siemens Healthineers, Erlangen, Germany) using a tube voltage of $100 \mathrm{kVp}$, a beam collimation of $128 \mathrm{~mm} \times 0.6 \mathrm{~mm}$, a pitch factor of 0.8 and a rotation time of $0.5 \mathrm{~s}$. Tube currents were defined to obtain four dose levels: $180 \mathrm{mAs}$ (D1.00, standard dose), $90 \mathrm{mAs}$ (D0.50, a twofold dose reduction), $45 \mathrm{mAs}$ (D0.25, VLD, a fourfold dose reduction) and $15 \mathrm{mAs}$ (D0.10, a tenfold dose reduction). The respective CTDIvol values were 7.02, 3.52, 1.75 and $0.59 \mathrm{mGy}$. The tube-current modulation system was disabled for each acquisition.

The raw data were reconstructed with the advanced model-based iterative reconstruction algorithm (ADMIRE) using the standard soft tissue reconstruction kernel (I30f). According to the Imactis ${ }^{\circledR}$ navigation system recommendations, the images were reconstructed at a slice thickness of $3.0 \mathrm{~mm}$ (at a $2.0 \mathrm{~mm}$ increment). A $350-\mathrm{mm}$ field of view was used to be close to the morphologies of both phantoms. A preliminary evaluation visually studied 

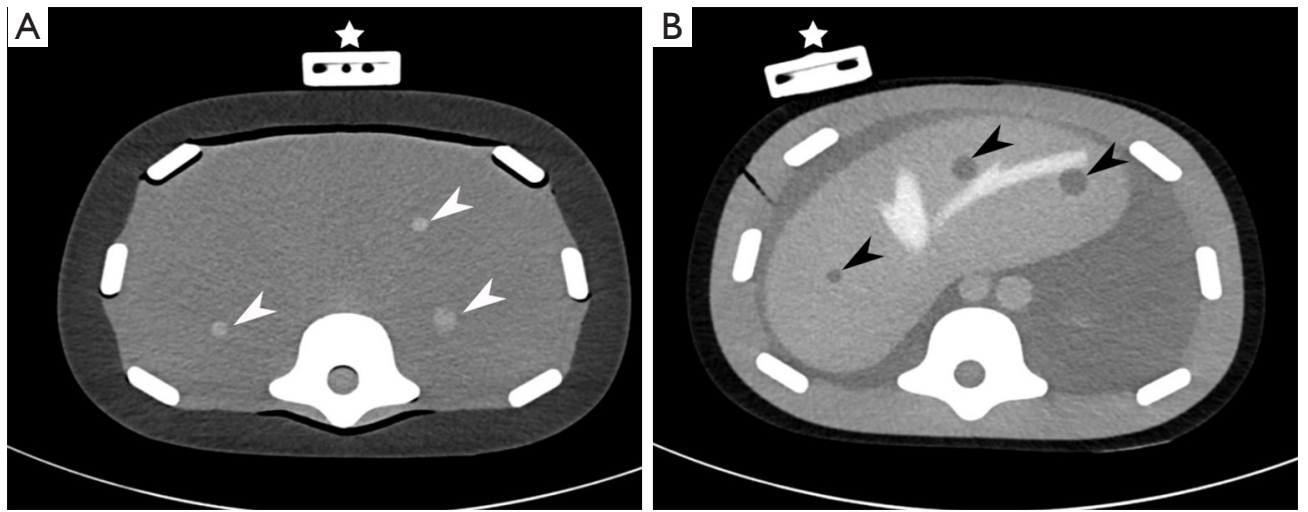

Figure 1 The two phantoms used in the study. Phantom 1 is depicted on the left (A) with hyperdense targets (white arrowhead) and the Imactis ${ }^{\circledR}$ magnetic transmitter (white asterisk). Phantom 2 is depicted on the right (B) with hypodense targets (black arrowhead).

Phantom 1

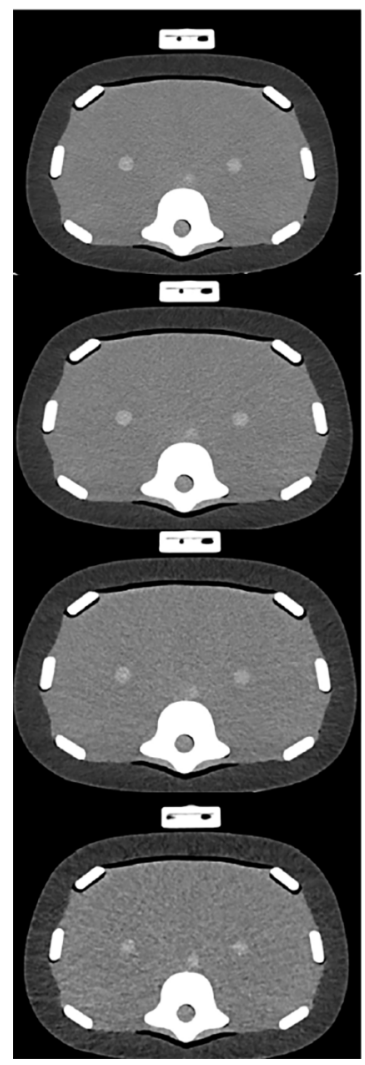

Phantom 2

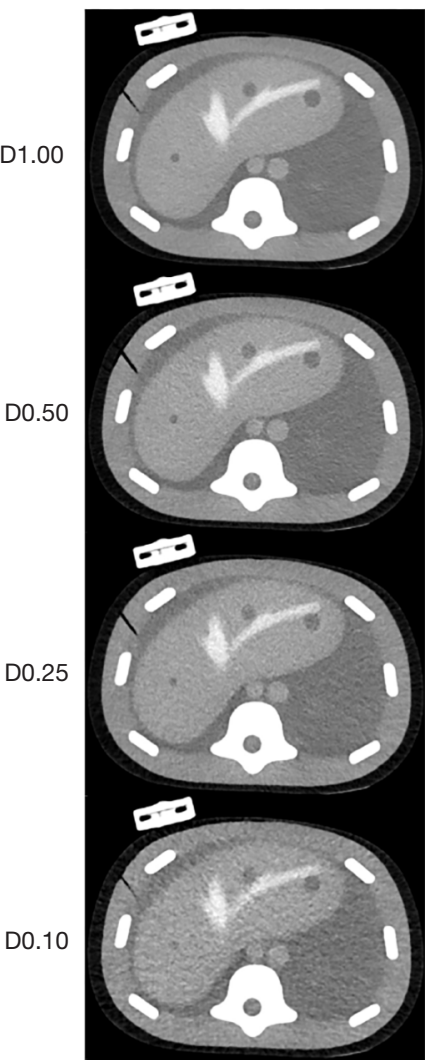

Figure 2 Phantoms with the four dose levels: D1.00 with ADMIRE 2, D0.50 with ADMIRE 3, D0.25 with ADMIRE 4 and D0.10 with ADMIRE 5. Left: phantom 1; right: phantom 2. D1.00: $180 \mathrm{mAs}$, $\mathrm{CTDI}_{\mathrm{vol}}=7.02 \mathrm{mGy}$; D0.50: $90 \mathrm{mAs}, \mathrm{CTDI}_{\mathrm{vol}}=3.52 \mathrm{mGy}$; D0.25: $45 \mathrm{mAs}, \mathrm{CTDI}_{\mathrm{vol}}=1.75 \mathrm{mGy}$; D0.10: $15 \mathrm{mAs}, \mathrm{CTDI}_{\mathrm{vol}}=0.59 \mathrm{mGy}$. ADMIRE, advanced model-based iterative reconstruction; $\mathrm{CTDI}_{\mathrm{vol}}$, volume computed tomography dose index. the influence of the ADMIRE levels on the different dose levels (Supplementary Appendix: section 1; Tables S1,S2, and Figure S1). ADMIRE Level 2 (A2) was used for D1.00, A3 for D0.50, A4 for D0.25 and A5 for D0.10 (Figure 2).

\section{Puncture procedure}

Two 12-mm diameter targets, one superficial $(6 \mathrm{~cm}$ below the skin) and one deep (at $12 \mathrm{~cm}$ ), were defined for each phantom. For each target, four puncture entry points were defined, two in the same plane (simple obliquity) and two out of plane (double obliquity with the angles in the $x$ and $\mathrm{z}$-axes both $>10^{\circ}$ ). Punctures were performed with a graduated needle with a 17-Ga coaxial introducer (Argon Medical device, Dallas, USA) using the Imactis ${ }^{\circledR}$ navigation system (Supplementary Appendix: section 2; Figure S2).

The punctures were performed by two radiologists: one was a senior radiologist (J Frandon) and the other was a junior radiologist ( $\mathrm{P}$ Akessoul). The senior radiologist (5 years of experience) had routinely used the Imactis ${ }^{\circledR}$ navigation system, but the junior radiologist (6 months of experience) had never used it. He received 1-hour basic theoretical training by the senior prior to the start of the study.

A total of 128 different scenarios were studied with different configurations: operator $(n=2)$, phantom $(n=2)$, target $(n=2)$, entry point $(n=4)$ and dose level $(n=4)$. The procedures were performed consecutively on each phantom after randomizing the order of the different scenarios. The procedures on the two phantoms were conducted during two different sessions. The operators were blinded to the dose levels.

Two acquisitions were performed for each procedure. After the prepuncture acquisition, the images were 


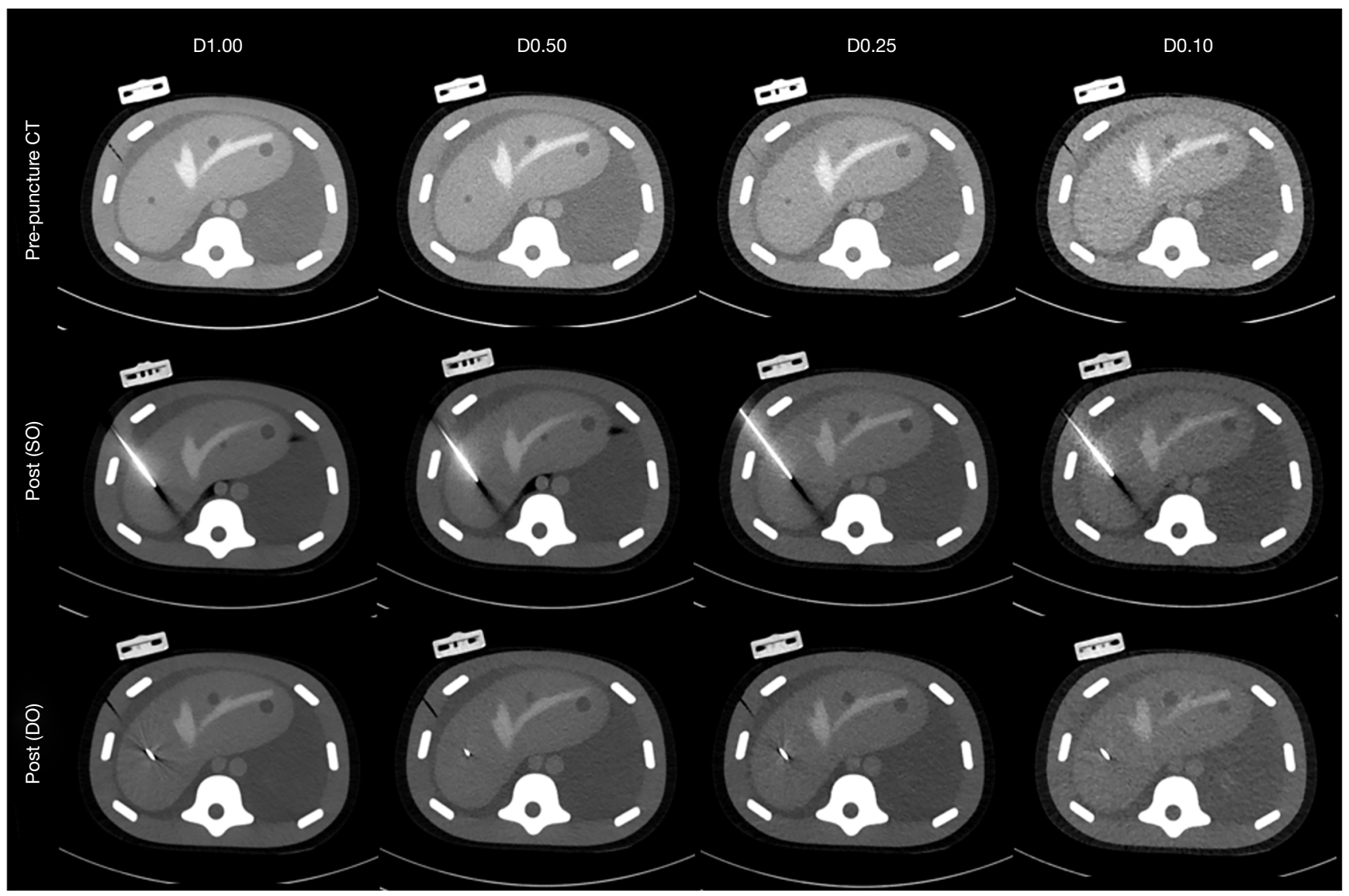

Figure 3 Example of simple obliquity (SO) and double obliquity (DO) punctures on phantom 2 with pre- and postprocedural CT at the four different dose levels. Windowing was adopted to show the needle tip. The artifacts differ between SO and DO punctures. D1.00: $180 \mathrm{mAs}$, $\mathrm{CTDI}_{\mathrm{vol}}=7.02 \mathrm{mGy}$; D0.50: $90 \mathrm{mAs} \mathrm{CTDI}_{\mathrm{vol}}=3.52 \mathrm{mGy}$; D0.25: $45 \mathrm{mAs}, \mathrm{CTDI}_{\mathrm{vol}}=1.75 \mathrm{mGy} ;$ D0.10: $15 \mathrm{mAs}, \mathrm{CTDI}_{\mathrm{vol}}=0.59 \mathrm{mGy}$. $\mathrm{CTDI}_{\mathrm{vol}}$, volume computed tomography dose index; DO, double obliquity; SO, simple obliquity.

transferred to Imactis ${ }^{\circledR}$ to plan the needle trajectory and evaluate its length. The puncture was then performed in one shot. The length of the needle was computed in the navigation system prior to the punction so that the needle length inserted was virtually followed without performing intermediate CT acquisition. A postpuncture control acquisition was performed to assess accuracy (Figure 3).

\section{Endpoints}

\section{Accuracy evaluation}

The accuracy of each procedure was evaluated by measuring the 3D Euclidean distance $(\mathrm{EuD})$, i.e., the 3D distance between the needle tip and the target isocenter (24). The distance was measured using Syngo.Via on a VB30 workstation (Siemens Healthineers, Erlangen, Germany) by one independent reader (E Bezandry) who was blinded to the procedure characteristics (operators, doses). If the target was not visible in the postprocedure acquisition because of artifacts, the preprocedure image was used with a needle projection and image fusion. The procedure duration was recorded only on phantom 2 .

\section{Objective analysis of image quality}

The objective image quality was analyzed using Syngo.Via on the VB30 workstation. For each acquisition, circular regions of interest (ROIs) were placed inside $\left(0.5 \mathrm{~cm}^{2}\right.$ diameter) and outside the target (background, $1 \mathrm{~cm}^{2}$ ). The mean attenuation (average number of pixels; HU value) and the image noise (standard deviation of pixels; $\sigma$ ) were computed for each ROI.

The contrast-to-noise ratio (CNR) between the target 
Table 1 Euclidean distance $(\mathrm{mm})$ between the isocenter of the 12-mm target and the tip of the needle as a function of operator experience, phantom used, depth and obliquity of the punctured target for each dose level

\begin{tabular}{|c|c|c|c|c|c|}
\hline Configuration & D1.00 & D0.50 & D0.25 & D0.10 & All \\
\hline Double obliquity & $6.8 \pm 3.3$ & $7.2 \pm 2.5$ & $7.9 \pm 3.5$ & $6.5 \pm 3.2$ & $7.1 \pm 3.1$ \\
\hline Superficial target & $6.0 \pm 2.1$ & $7.8 \pm 3.6$ & $7.9 \pm 2.7$ & $7.4 \pm 2.7$ & $7.3 \pm 2.9$ \\
\hline Deep target & $8.2 \pm 4.2$ & $6.4 \pm 2.5$ & $6.5 \pm 3.2$ & $5.7 \pm 2.3$ & $6.7 \pm 3.2$ \\
\hline Phantom 2 & $6.6 \pm 2.8$ & $6.5 \pm 2.4$ & $6.1 \pm 2.4$ & $6.3 \pm 2.7$ & $6.4 \pm 2.5$ \\
\hline Senior & $6.8 \pm 3.1$ & $7.6 \pm 2.7$ & $7.2 \pm 2.6$ & $6.4 \pm 2.0$ & $7.0 \pm 2.6$ \\
\hline Junior & $7.4 \pm 3.9$ & $6.7 \pm 3.5$ & $7.2 \pm 3.4$ & $6.7 \pm 3.2$ & $7.0 \pm 3.5$ \\
\hline All & $7.1 \pm 3.5$ & $7.1 \pm 3.1$ & $7.2 \pm 3.0$ & $6.6 \pm 2.6$ & $7.0 \pm 3.1$ \\
\hline
\end{tabular}

Values are expressed as mean \pm standard deviation. D1.00: $180 \mathrm{mAs}, \mathrm{CTDI}_{\mathrm{vol}}=7.02 \mathrm{mGy}$; D0.50: $90 \mathrm{mAs} \mathrm{CTDI}_{\mathrm{vol}}=3.52 \mathrm{mGy}$; D0.25: $45 \mathrm{mAs}, \mathrm{CTDI}_{\text {vol }}=1.75 \mathrm{mGy}$; D0.10: $15 \mathrm{mAs}$, CTDI $_{\text {vol }}=0.59 \mathrm{mGy}$. CTDI ${ }_{\text {vol }}$, volume computed tomography dose index.

and the background was calculated as follows:

$$
C N R=\frac{\left|H U_{\text {ROI background }}-H U_{\text {ROI target }}\right|}{\sqrt{\frac{\sigma_{\text {ROI background }}^{2}+\sigma_{\text {ROI target }}^{2}}{2}}}
$$

Subjective image quality and confidence level analysis

Immediately after each puncture, both radiologists assessed the subjective image quality on Imactis ${ }^{\circledR}$ using the following scale: $1=$ target difficult to detect on the two orthogonal plans reconstructed by Imactis ${ }^{\circledR} ; 2=$ target difficult to detect on one of the two plans; $3=$ target detectable on the two plans, with intermediate noise; $4=$ target perfectly detectable on 1 of the 2 plans; 5 = target perfectly detectable on both plans. A score $\geq 3$ was considered sufficient to provide good guidance. Radiologists also assessed their confidence levels after the procedure as follows: $1=$ target probably not reached; $2=$ target possibly reached; $3=$ target probably reached; $4=$ target most likely reached; $5=$ target reached with certainty. A confidence level $\geq 4$ reflected good confidence in the procedure.

\section{Statistical analysis}

Data processing and analysis were conducted with $\mathrm{R}$ (v3.20) and RStudio ( $\mathrm{v} 0.98 .113$ ). The variables are presented as means and standard deviations. They were compared using paired Student's $t$-tests for parametric variables, MannWhitney Wilcoxon tests for nonparametric variables and ANOVA for multiple parametric variables. P values below
0.05 were considered statistically significant. For subjective data, the interoperator agreement for each protocol was computed by Cohen's kappa (poor, $\kappa=0.00-0.20$; fair $\kappa=0.21-0.40$; moderate $\kappa=0.41-0.60$, good, $\kappa=0.61-0.80$; excellent, $\kappa=0.81-1.00$ ).

\section{Results}

\section{Feasibility of the protocol}

The Imactis ${ }^{\circledR}$ navigation system functioned properly at all the different dose levels and provided accurate virtual guidance in the $3 \mathrm{D}$ volume. The targets were visible in all the preprocedural acquisitions.

However, the target was not visible in 8 of the postpuncture acquisitions $(8 / 128,6.3 \%)$ because of needle artifacts; of these, 5 occurred using D0.10 (5/32; 15.6\%) and 3 using D0.25 (3/32; 9.4\%) (Figure 3).

\section{Accuracy evaluation of punctures with dose reduction}

The mean $\mathrm{EuD}$ was $7.0 \pm 3.1 \mathrm{~mm}$ for the 128 punctures performed. The puncture accuracy was not altered by the dose reduction: the mean $\mathrm{EuD}$ values were $7.1 \pm 3.5 \mathrm{~mm}$ for D1.00, 7.1 $\pm 3.1 \mathrm{~mm}$ for D0.50, 7.2 $\pm 3.0 \mathrm{~mm}$ for D0.25 and $6.6 \pm 2.6 \mathrm{~mm}$ for D0.10 (Table 1 and Figure 4).

\section{Punctures accuracy under different scenarios}

Operator experience, obliquity and depth had no significant 


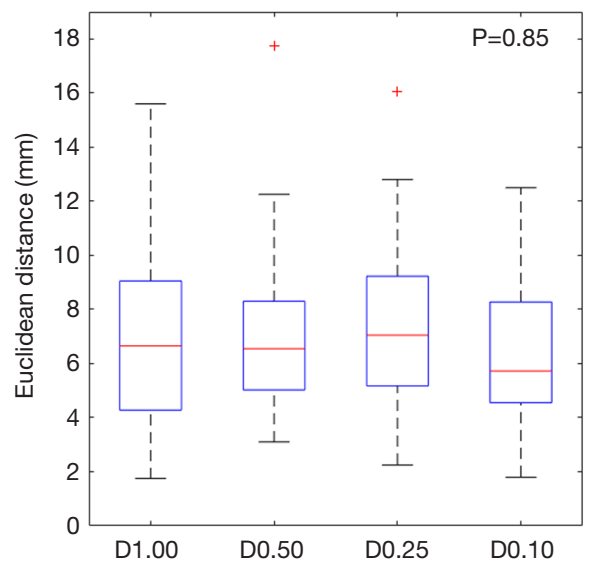

Figure 4 Boxplot of the mean Euclidean distance $(\mathrm{mm})$ between the isocenter of the $12 \mathrm{~mm}$ target and the tip of the needle according to the protocol used. D1.00: $180 \mathrm{mAs} \mathrm{CTDI}_{\mathrm{vol}}=7.02 \mathrm{mGy}$; D0.50: $90 \mathrm{mAs}, \mathrm{CTDI}_{\mathrm{vol}}=3.52 \mathrm{mGy}$; D0.25: $45 \mathrm{mAs}, \mathrm{CTDI}_{\mathrm{vol}}=1.75 \mathrm{mGy}$; D0.10: $15 \mathrm{mAs} \mathrm{CTDI}_{\mathrm{vol}}=0.59 \mathrm{mGy}$. $\mathrm{P}$ value lower than 0.05 was considered significant. CTDI ${ }_{\text {vol }}$, volume computed tomography dose index.

effect on puncture accuracy, regardless of the dose level (Figure 5). The punctures were significantly more accurate when performed on phantom 2 than on phantom $1(6.4 \pm 2.5$ vs. $7.6 \pm 3.4 \mathrm{~mm}, \mathrm{P}=0.02)$. However, no differences occurred between the EuDs reported for the first 10 and last 10 procedures on each phantom.

The mean procedure duration was not significantly different between the different dose levels, although there was a tendency toward longer times under reduced doses. Mean procedure durations were $39 \pm 12 \mathrm{~s}$ for D1.00 dose, $36 \pm 14 \mathrm{~s}$ for D0.50, $43 \pm 22 \mathrm{~s}$ for D0.25 and $59 \pm 28 \mathrm{~s}$ for D0.10 ( $\mathrm{P}=0.052)$. The junior operator spent significantly more time on each puncture (mean procedure duration of $51 \pm 25 \mathrm{~s}$ ) than did the senior operator (mean procedure duration of $38 \pm 14 \mathrm{~s}, \mathrm{P}=0.02$ ) (Figure 6).

\section{Objective analysis of image quality}

The mean attenuation of the target and background were not significantly different for all dose levels on phantom 2 , but it was significant for the targets on the phantom $(\mathrm{P}<0.001)$. Noise increased with dose reduction and was significantly higher $(\mathrm{P}<0.001)$ for $\mathrm{D} 0.10$ than for $\mathrm{D} 1.00$ for both targets and background. The CNR decreased with dose reduction and was significantly lower for D0.10 than for D1.00 ( $\mathrm{P}<0.001)$ (from $-43 \%$ to $-36 \%$ ) (Table 2$)$.

\section{Subjective image quality and confidence level analysis}

Both the image quality and confidence level decreased with dose reduction for both operators. Overall, the junior operator tended to rate both the image quality and confidence level at lower values than did the senior operator. For D1.00, the image quality was scored as $\geq 3$ for $100 \%$ of procedures for both radiologists; however, for D0.10, image quality reached $\geq 3$ in only $44 \%$ of the procedures by the senior and $19 \%$ by the junior.

For D1.00, the confidence level was scored as $\geq 4$ for $100 \%$ of procedures by the senior and $56 \%$ by the junior; however, for $\mathrm{D} 0.10$ it reached only for $13 \%$ and $0 \%$ of procedures for the senior and the junior, respectively (Table 3).

The interobserver agreement for all dose levels was good for both image quality evaluation $(\kappa=0.795,95 \%$ CI: $0.667-0.924)$ and for confidence level $(\kappa=0.778,95 \% \mathrm{CI}$ : 0.635-0.922); however, it decreased with dose reduction. For D1.00, the interobserver agreements were excellent for image quality and good for confidence level, but they were moderate for these two criteria for D0.10 (Table 3).

\section{Discussion}

Computer-assisted CT-guided puncture was both feasible and accurate with no significant differences at different dose reduction protocols from D1.00 to D0.10, in which the dose was reduced by $91.6 \%$. The experiment showed no influence from operator experience, obliquity path or target depth on the accuracy. However, the operators felt significantly less confident for D0.10.

Many CT navigation systems exist that provide precise puncture locations to help guide radiologists (15). Imactis ${ }^{\circledR}$ is one of the easiest and most ergonomic to use; unlike many other electromagnetic guidance systems it provides real-time needle tracking (25-27). In contrast, laser (28) and optical guidance (25) systems are limited because they require a direct line between the markers and the detection system. However, one drawback of Imactis ${ }^{\circledR}$ is that the use of helicoidal acquisition is mandatory. Indeed, optimization recommendations involve limiting the length and number of helicoidal acquisitions and preferring sequential or fluoroscopic acquisition when possible (1,11-13). The greatest exposures occur during plan acquisition and final control, where helical mode contributed $82 \%$ of the total dose length product (DLP) in a recent multicentric study (13). Some studies have proposed adapting mAs and $\mathrm{kVp}$ to patient size and diameter to help reduce the dose 

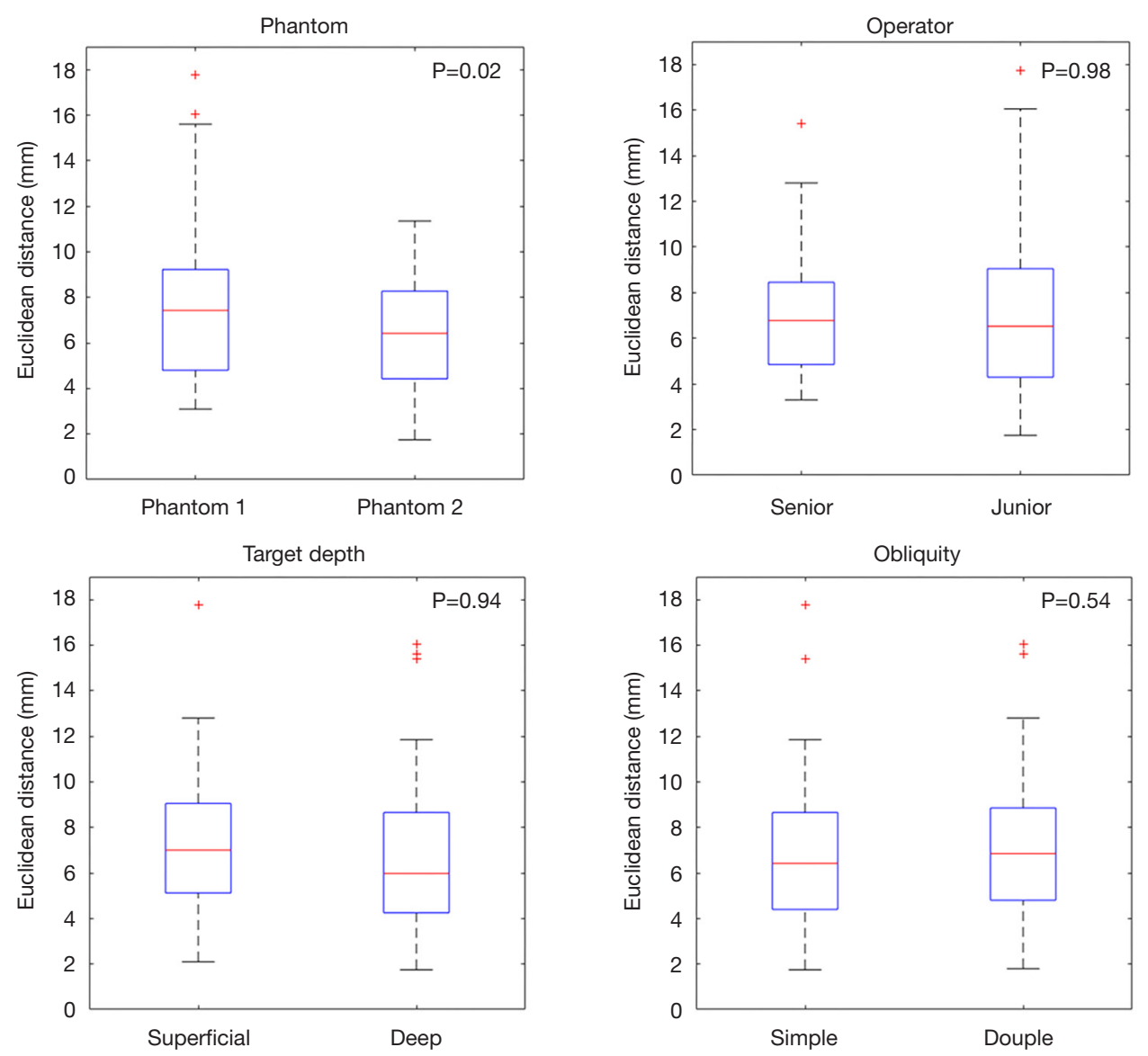

Figure 5 Boxplot of the mean Euclidean distance $(\mathrm{mm})$ between the isocenter of the $12 \mathrm{~mm}$ target and the tip of the needle as a function of obliquity, target depth, phantom and operator experience. $\mathrm{P}$ value lower than 0.05 was considered significant.

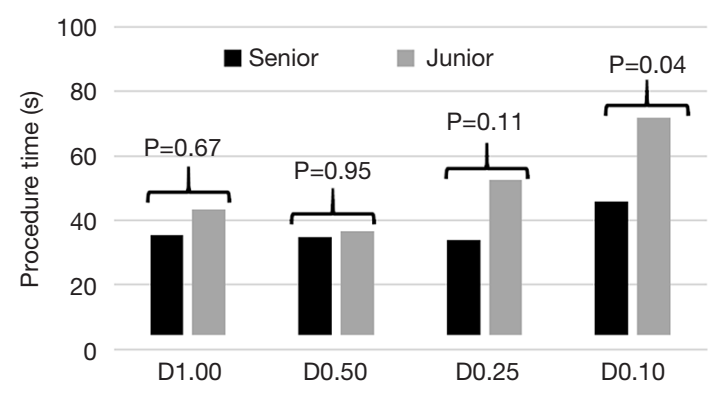

Figure 6 Mean procedure time (s) for both operators according to the protocol used. D1.00: $180 \mathrm{mAs}, \mathrm{CTDI}_{\mathrm{vol}}=7.02 \mathrm{mGy}$; D0.50: $90 \mathrm{mAs}, \mathrm{CTDI}_{\mathrm{vol}}=3.52 \mathrm{mGy}$; D0.25: $45 \mathrm{mAs}, \mathrm{CTDI}_{\mathrm{vol}}=1.75 \mathrm{mGy}$; D0.10: $15 \mathrm{mAs} \mathrm{CTDI}_{\mathrm{vol}}=0.59 \mathrm{mGy}$. P value lower than 0.05 was considered significant. CTDI ${ }_{\mathrm{vol}}$, volume computed tomography dose index.
$(12,29)$. Lucey et al. evaluated the feasibility of a low-dose protocol for CT control during the procedure with current reductions of up to $30 \mathrm{mAs}$ but with no modifications to $\mathrm{kVp}(120-140)$ (30). The purpose of this study was to optimize our interventional protocols in the same way to investigate how far dose reduction can go without altering the puncture accuracy. The tube voltage was not modified; it was fixed to $100 \mathrm{kVp}$ to be coherent with abdominal puncture. However, the tube current was reduced from 180 to $15 \mathrm{mAs}$ to reduce the dose level (the CTDIvol ranged from 7.02 to $0.59 \mathrm{mGy}$ ). Different levels of iterative reconstruction were defined to maintain sufficient image quality to aid in punctures. Alagic et al. also demonstrated that iterative reconstruction could help reduce the dose level for musculoskeletal biopsies (8). 
Table 2 Quantitative analysis and comparison between the four dose levels

\begin{tabular}{|c|c|c|c|c|c|c|c|}
\hline $\begin{array}{l}\text { Objective } \\
\text { image quality }\end{array}$ & Parameters & D1.00 & D0.50 & D0.25 & D0.10 & $\begin{array}{c}\text { Difference } \\
\text { [D0.10 vs. D1.00 (\%)] }\end{array}$ & $\begin{array}{c}\text { P value } \\
\text { (D0.10 vs. D1.00) }\end{array}$ \\
\hline \multirow{2}{*}{$\begin{array}{l}\text { Mean } \\
\text { attenuation } \\
(\mathrm{HU})\end{array}$} & Targets, phantom 1 & $81.5 \pm 6$ & $80.5 \pm 3.5$ & $77.6 \pm 1.9$ & $69.1 \pm 4.5$ & -15 & $<0.001$ \\
\hline & Targets, phantom 2 & $31.4 \pm 3.2$ & $31.5 \pm 3.2$ & $31.2 \pm 5.2$ & $30.9 \pm 3.2$ & -2 & 0.62 \\
\hline \multirow{3}{*}{ Noise $(\mathrm{HU})$} & Background, phantom 1 & $9.5 \pm 0.7$ & $9.6 \pm 1.4$ & $11.8 \pm 1.4$ & $15.0 \pm 1.4$ & 58 & $<0.001$ \\
\hline & Targets, phantom 2 & $7.1 \pm 1.0$ & $9.9 \pm 1.0$ & $10.8 \pm 1.4$ & $11.1 \pm 2.2$ & 58 & $<0.001$ \\
\hline & Background, phantom 2 & $8.1 \pm 1.0$ & $10.5 \pm 0.8$ & $11.0 \pm 0.9$ & $13.8 \pm 1.0$ & 69 & $<0.001$ \\
\hline
\end{tabular}

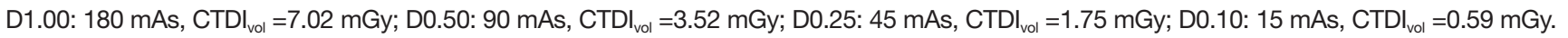
$\mathrm{CTDI}_{\mathrm{vol}}$, volume computed tomography dose index; CNR, contrast-to-noise ratio; HU, Hounsfield unit.

Table 3 Subjective evaluation of image quality and confidence level

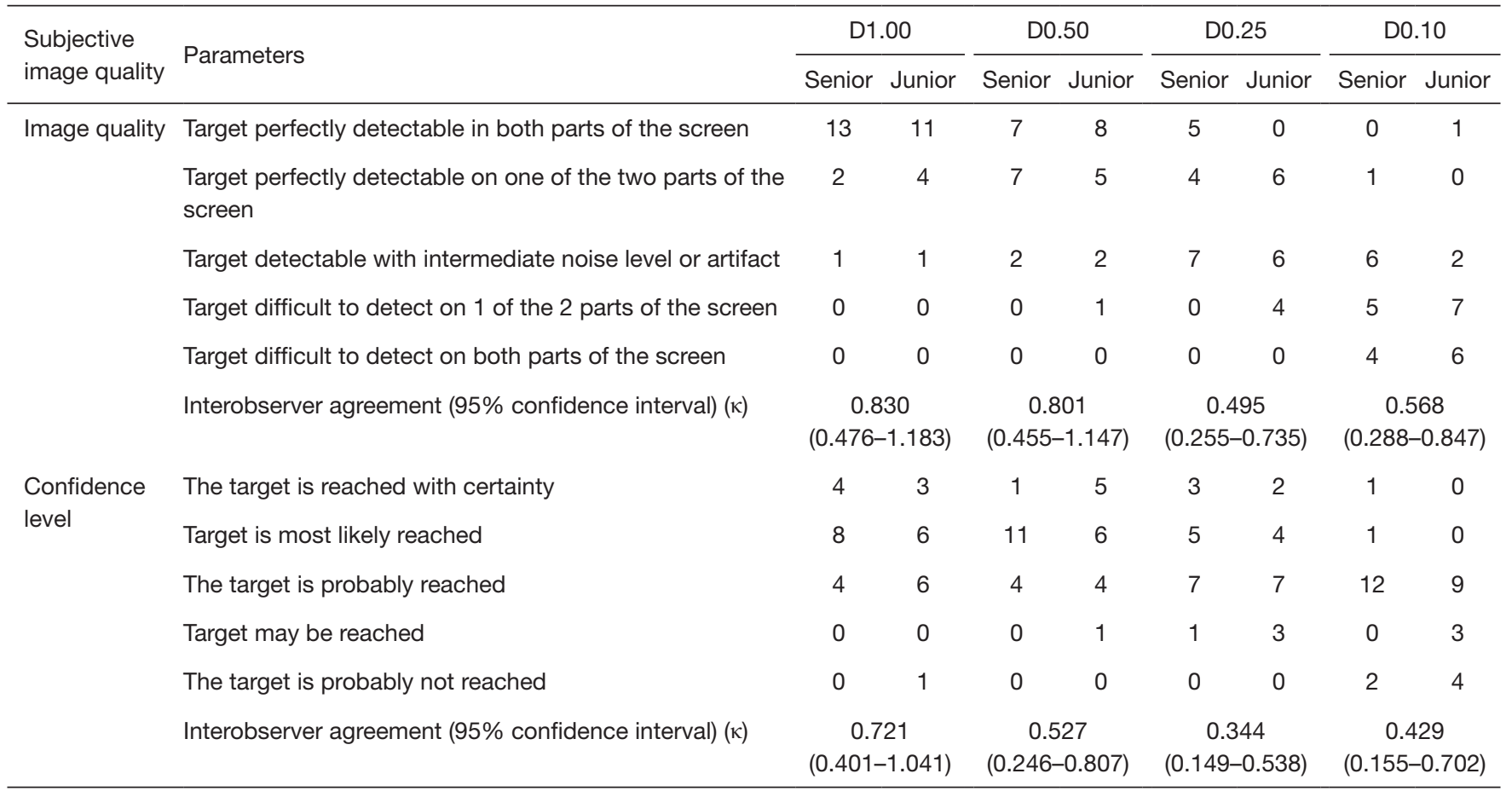

D1.00: $180 \mathrm{mAs}$, CTDI $_{\mathrm{vol}}=7.02 \mathrm{mGy}$; D0.50: $90 \mathrm{mAs}, \mathrm{CTDI}_{\mathrm{vol}}=3.52 \mathrm{mGy}$; D0.25: $45 \mathrm{mAs}, \mathrm{CTDI}_{\mathrm{vol}}=1.75 \mathrm{mGy}$; D0.10: 15 mAs, CTDI $=0.59 \mathrm{mGy}$. CTDI $_{\text {vol }}$, volume computed tomography dose index.

The present study found that accuracy was not dependent on operator experience or trajectory difficulty, as was demonstrated in a randomized trial studying Imactis ${ }^{\circledR}$ precision (17). This showed for the first time that the dose, even a dose reduction of $-92 \%$ compared to standard protocol, does not alter the accuracy or risk of punctures. 
Accuracy was lower than in a study by Durand et al. (7.0 vs. $4.1 \mathrm{~mm}$ ) (17), which may be due to the different methods of measurement used here [EuD (31)] and the one-shot protocol with $100 \%$ virtual guidance and no intermediate control to adapt the needle position. Indeed, Imactis ${ }^{\circledR}$ is a passive guidance system and potential movement of the operator during the insertion cannot be corrected without intermediate control. The punctures were significantly less accurate when using phantom 1 than when using phantom 2 (7.6 $\pm 3.4 v s$. $6.4 \pm 2.5 \mathrm{~mm}, \mathrm{P}=0.02$ ), which could be related to the composition of phantom 1 (dense targets in a monobloc of soft Zerdine ${ }^{\circledR}$ ) and reciprocal movements between the needle and target. The second phantom was closer to reality with a homogenous dense structure and a filling inside representing the target.

Although the accuracy was not altered by dose reduction, subjective image quality and operator confidence levels were not as good with D0.10, with ratings of $56 \%$ for sufficient quality and only $13 \%$ for confidence. The evaluations were worse for the junior, who rated sufficient image quality at only $19 \%$ and rated the confidence at $0 \%$ with D0.10. This difference could be reduced if the time spent performing the puncture were longer with D0.10. However, the accuracy was the same, showing the safety of the procedure with careful puncture. This result was also found in another study evaluating subjective image quality with D0.10 acquisition $(21,22)$. This result emphasizes the necessity for changing the operator's mind, switching the operator's expectation from a "good" image to a "sufficient" image. The radiologist would need to learn to be comfortable with a sufficient image quality that allows precise puncture. It also emphasizes the necessity for developing specific conditions in which trainees can learn to work with D0.10 images.

This study shows the feasibility of ultra-low-dose puncture thanks to the use of an iterative reconstruction algorithm and it can serve as guidance for a further human study. The D0.10 protocol can be a good benchmark to begin the procedure. The advantage of Imactis ${ }^{\circledR}$ is that it is an external navigation system that can be stopped or switched to a higher dose level at any moment. Indeed, Lucey et al., in their low-dose protocol, which did not use iterative reconstruction, found that the target was not visualized in 13 of 201 patients, who required novel acquisition under a higher dose (30). All the targets were visible on the prepuncture acquisitions in our study, but we can imagine a similar protocol in clinical practice with a switch to D0.25 or D0.50 if D0.10 did not allow the target to be seen.
The study has some limitations, mainly due to its design and the use of phantoms that cannot reproduce real situations (e.g., respiratory movement, height and weight differences, need to avoid perforation of small blood vessels and bile ducts). The stereotypic punctures used here could lead to a learning curve despite the randomization of the scenarios. Moreover, some permanent tracking might be evident if debris and air bubbles were entrained in the gel during the biopsy procedure. Although we found no differences in accuracy between the first and last punctures, we cannot exclude the possibility of a learning bias. Stereotypic targets cannot replace clinical practice, in which lesions could be isodense and thus less visible. However, it has been shown that D0.10 images can be used to detect hypodense lesions with precision in abdominal imaging (21). The punctures were performed on a single CT scan, so the protocol defined could only be transferrable to CT scans using a similar iterative reconstruction algorithm. The targets could not be seen due to needle artifacts in $15.6 \%$ of the D0.10 postprocedural acquisitions, which could be challenging to iterative control in clinical use. Last, a baseline navigation error is associated with this specific needle guidance system. The results found in this study may not apply to other navigation systems.

\section{Conclusions}

The computer-assisted CT-guided procedures were precise regardless of the dose, trajectory obliquity, target depth, or operator experience. Using 2 anthropomorphic phantoms, this study showed the feasibility of performing punctures even under dramatic dose reduction. The study investigated using different levels of iterative reconstruction, from a standard dose to $-91.6 \%$ dose reduction (D0.10). Puncture accuracy was unaffected and was the same under all dose reduction protocols, with either a junior or senior operator, mono- or double obliquity paths, and superficial or deep targets. However, with $-91.6 \%$ dose reduction (D0.10) operator confidence in the procedure was reduced, and clinical evaluations were performed.

\section{Acknowledgments}

The authors thank Dr. Hélène de Forges for her help and her participation in the study. They also thank the Clinical Research Department of the Nîmes University Hospital and the BESPIM unit for their methodologic, regulatory and administrative support throughout the study. 
Funding: The study was funded by an internal Nîmes University Hospital call for projects, NIMAO, funding number NIMAO/2018-02/JF-01.

\section{Footnote}

Conflicts of Interest: All authors have completed the ICMJE uniform disclosure form (available at https://dx.doi. org/10.21037/qims-21-328). Dr. RL serves as an unpaid deputy editor of Quantitative Imaging in Medicine and Surgery. The other authors have no conflicts of interest to declare.

Ethical Statement: The authors are accountable for all aspects of the work in ensuring that questions related to the accuracy or integrity of any part of the work are appropriately investigated and resolved. The study was conducted only on phantoms, no patient was included. Ethical approval and written informed consent are not applicable for this study.

Open Access Statement: This is an Open Access article distributed in accordance with the Creative Commons Attribution-NonCommercial-NoDerivs 4.0 International License (CC BY-NC-ND 4.0), which permits the noncommercial replication and distribution of the article with the strict proviso that no changes or edits are made and the original work is properly cited (including links to both the formal publication through the relevant DOI and the license). See: https://creativecommons.org/licenses/by-nc-nd/4.0/.

\section{References}

1. Chintapalli KN, Montgomery RS, Hatab M, Katabathina VS, Guiy K. Radiation dose management: part 1, minimizing radiation dose in CT-guided procedures. AJR Am J Roentgenol 2012;198:W347-51.

2. Guberina N, Forsting M, Ringelstein A, Suntharalingam S, Nassenstein K, Theysohn J, Wetter A. Radiation exposure during CT-guided biopsies: recent CT machines provide markedly lower doses. Eur Radiol 2018;28:3929-35.

3. Kallianos KG, Elicker BM, Henry TS, Ordovas KG, Nguyen J, Naeger DM. Instituting a Low-dose CT-guided Lung Biopsy Protocol. Acad Radiol 2016;23:1130-6.

4. Kloeckner R, dos Santos DP, Schneider J, Kara L, Dueber C, Pitton MB. Radiation exposure in CT-guided interventions. Eur J Radiol 2013;82:2253-7.

5. Leng S, Christner JA, Carlson SK, Jacobsen M, Vrieze
TJ, Atwell TD, McCollough CH. Radiation dose levels for interventional CT procedures. AJR Am J Roentgenol 2011;197:W97-103.

6. Levesque VM, Shyn PB, Tuncali K, Tatli S, Nawfel RD, Olubiyi O, Silverman SG. Radiation dose during CTguided percutaneous cryoablation of renal tumors: Effect of a dose reduction protocol. Eur J Radiol 2015;84:2218-21.

7. Paik NC. Radiation dose reduction in CT fluoroscopyguided lumbar interlaminar epidural steroid injection by minimizing preliminary planning imaging. Eur Radiol 2014:24:2109-17.

8. Alagic Z, Alagic H, Bujila R, Srivastava S, Jasim S, Lindqvist $M$, Wick MC. First experiences of a lowdose protocol for CT-guided musculoskeletal biopsies combining different radiation dose reduction techniques. Acta Radiol 2020;61:28-36.

9. Weir VJ, Zhang J, Bruner AP. Impact of physician practice on patient radiation dose during CT guided biopsy procedures. J Xray Sci Technol 2014;22:309-19.

10. Greffier J, Pereira FR, Viala P, Macri F, Beregi JP, Larbi A. Interventional spine procedures under CT guidance: How to reduce patient radiation dose without compromising the successful outcome of the procedure? Phys Med 2017;35:88-96.

11. Lamba R, Corwin MT, Fananapazir G. Practical dose reduction tips for abdominal interventional procedures using CT-guidance. Abdom Radiol (NY) 2016;41:743-53.

12. Sarti M, Brehmer WP, Gay SB. Low-dose techniques in CT-guided interventions. Radiographics 2012;32:1109-19; discussion 1119-20.

13. Greffier J, Ferretti G, Rousseau J, Andreani O, Alonso E, Rauch A, et al. National dose reference levels in computed tomography-guided interventional procedures-a proposal. Eur Radiol 2020;30:5690-701.

14. Joemai RM, Zweers D, Obermann WR, Geleijns J. Assessment of patient and occupational dose in established and new applications of MDCT fluoroscopy. AJR Am J Roentgenol 2009;192:881-6.

15. Arnolli MM, Hanumara NC, Franken M, Brouwer DM, Broeders IA. An overview of systems for CT- and MRIguided percutaneous needle placement in the thorax and abdomen. Int J Med Robot 2015;11:458-75.

16. Wang D, Ma D, Wong ML, Wáng YX. Recent advances in surgical planning \& navigation for tumor biopsy and resection. Quant Imaging Med Surg 2015;5:640-8.

17. Durand P, Moreau-Gaudry A, Silvent AS, Frandon J, Chipon E, Médici M, Bricault I. Computer assisted electromagnetic navigation improves accuracy in 
computed tomography guided interventions: A prospective randomized clinical trial. PLoS One 2017;12:e0173751.

18. Teriitehau C, Rabeh H, Pessis E, Sénéchal Q, Besse $F$, Bravetti $M$. Reduction of patient radiation dose during percutaneous CT vertebroplasty: Impact of a new computer-assisted navigation (CAN) system. Radioprotection 2020;5 5:11-6.

19. Patino M, Fuentes JM, Singh S, Hahn PF, Sahani DV. Iterative Reconstruction Techniques in Abdominopelvic CT: Technical Concepts and Clinical Implementation. AJR Am J Roentgenol 2015;205:W19-31.

20. Greffier J, Fernandez A, Macri F, Freitag C, Metge L, Beregi JP. Which dose for what image? Iterative reconstruction for CT scan. Diagn Interv Imaging 2013;94:1117-21.

21. Larbi A, Orliac C, Frandon J, Pereira F, Ruyer A, Goupil J, Macri F, Beregi JP, Greffier J. Detection and characterization of focal liver lesions with ultra-low dose computed tomography in neoplastic patients. Diagn Interv Imaging 2018;99:311-20.

22. Macri F, Greffier J, Pereira F, Rosa AC, Khasanova E, Claret PG, Larbi A, Gualdi G, Beregi JP. Value of ultralow-dose chest CT with iterative reconstruction for selected emergency room patients with acute dyspnea. Eur J Radiol 2016;85:1637-44.

23. Lu X, Lu Z, Yin J, Gao Y, Chen X, Guo Q. Effects of radiation dose levels and spectral iterative reconstruction levels on the accuracy of iodine quantification and virtual monochromatic CT numbers in dual-layer spectral detector CT: an iodine phantom study. Quant Imaging Med Surg 2019;9:188-200.

24. Putzer D, Arco D, Schamberger B, Schanda F, Mahlknecht J, Widmann G, Schullian P, Jaschke W, Bale R.

Comparison of Two Electromagnetic Navigation Systems For CT-Guided Punctures: A Phantom Study. Rofo

Cite this article as: Frandon J, Akessoul P, Hamard A, Bezandry E, Loffroy R, Addala T, Bertrand MM, Beregi JP, Greffier J. Comparison of acquisition and iterative reconstruction parameters in abdominal computed tomographyguided procedures: a phantom study. Quant Imaging Med Surg 2022;12(1):281-291. doi: 10.21037/qims-21-328
2016;188:470-8.

25. Glossop ND. Advantages of optical compared with electromagnetic tracking. J Bone Joint Surg Am 2009;91 Suppl 1:23-8.

26. Grand DJ, Atalay MA, Cronan JJ, Mayo-Smith WW, Dupuy DE. CT-guided percutaneous lung biopsy: comparison of conventional CT fluoroscopy to CT fluoroscopy with electromagnetic navigation system in 60 consecutive patients. Eur J Radiol 2011;79:e133-6.

27. Zhang Z, Shao G, Zheng J, Wen S, Zeng H, Hao W, Luo J, Guo L. Electromagnetic navigation to assist with computed tomography-guided thermal ablation of liver tumors. Minim Invasive Ther Allied Technol 2020;29:275-82.

28. Moser C, Becker J, Deli M, Busch M, Boehme M, Groenemeyer DH. A novel Laser Navigation System reduces radiation exposure and improves accuracy and workflow of CT-guided spinal interventions: a prospective, randomized, controlled, clinical trial in comparison to conventional freehand puncture. Eur J Radiol 2013;82:627-32.

29. Tam AL, Ensor JE, Zvavanjanja RC, Philip A, Abdelsalam ME, Huang SY, Gupta S, Jones AK. JOURNAL CLUB: Standardizing CT-Guided Biopsy Procedures: Patient Dose and Image Noise. AJR Am J Roentgenol 2015;205:W390-9.

30. Lucey BC, Varghese JC, Hochberg A, Blake MA, Soto JA. CT-guided intervention with low radiation dose: feasibility and experience. AJR Am J Roentgenol 2007;188:1187-94.

31. Bot M, van den Munckhof P, Bakay R, Stebbins G, Verhagen Metman L. Accuracy of Intraoperative Computed Tomography during Deep Brain Stimulation Procedures: Comparison with Postoperative Magnetic Resonance Imaging. Stereotact Funct Neurosurg 2017;95:183-8. 
1. Selection of the advanced model-based iterative reconstruction (ADMIRE) levels (Tables S1,S2, Figure S1)

Before starting the experiments, a test was performed on the fantom 1 consisting in carrying out multiple acquisitions by varying the constants (mAs and $\mathrm{Kv}$ ) and the levels of iterative reconstructions (ADMIRE).

The images were visually analyzed by the two operators to determine in consensus what was the best level of ADMIRE for each dose level to allow sufficient detection of the targets.

Table S1 Acquisition parameters for each dose level

\begin{tabular}{lcccc}
\hline Acquisition & $\mathrm{kV}$ & $\mathrm{mAs}$ & CTDI $_{\text {vol }}(\mathrm{mGy})$ & $\mathrm{DLP}(\mathrm{mGy} \cdot \mathrm{cm})$ \\
\hline D0.10 & 100 & 15 & 0.59 & 10.62 \\
D0.25 & 100 & 45 & 1.75 & 31.50 \\
D0.50 & 100 & 90 & 3.52 & 63.36 \\
D1.00 & 100 & 180 & 7.02 & 126.36
\end{tabular}

D1.00 corresponds to standard dose. The tube voltage was fixed to $100 \mathrm{kVp}$. DLP, dose length product; CTDIvol, volume computed tomography dose index.

Table S2 ADMIRE levels corresponding to the dose levels

\begin{tabular}{lccc}
\hline Acquisition & ADMIRE & ADMIRE & ADMIRE \\
\hline D 0.10 & 3 & 4 & 5 \\
D 0.25 & 3 & 4 & 5 \\
D 0.50 & 0 & 2 & 3 \\
D 1.00 & & 0 & 2 \\
\hline
\end{tabular}

ADMIRE, advanced model-based iterative reconstruction. 


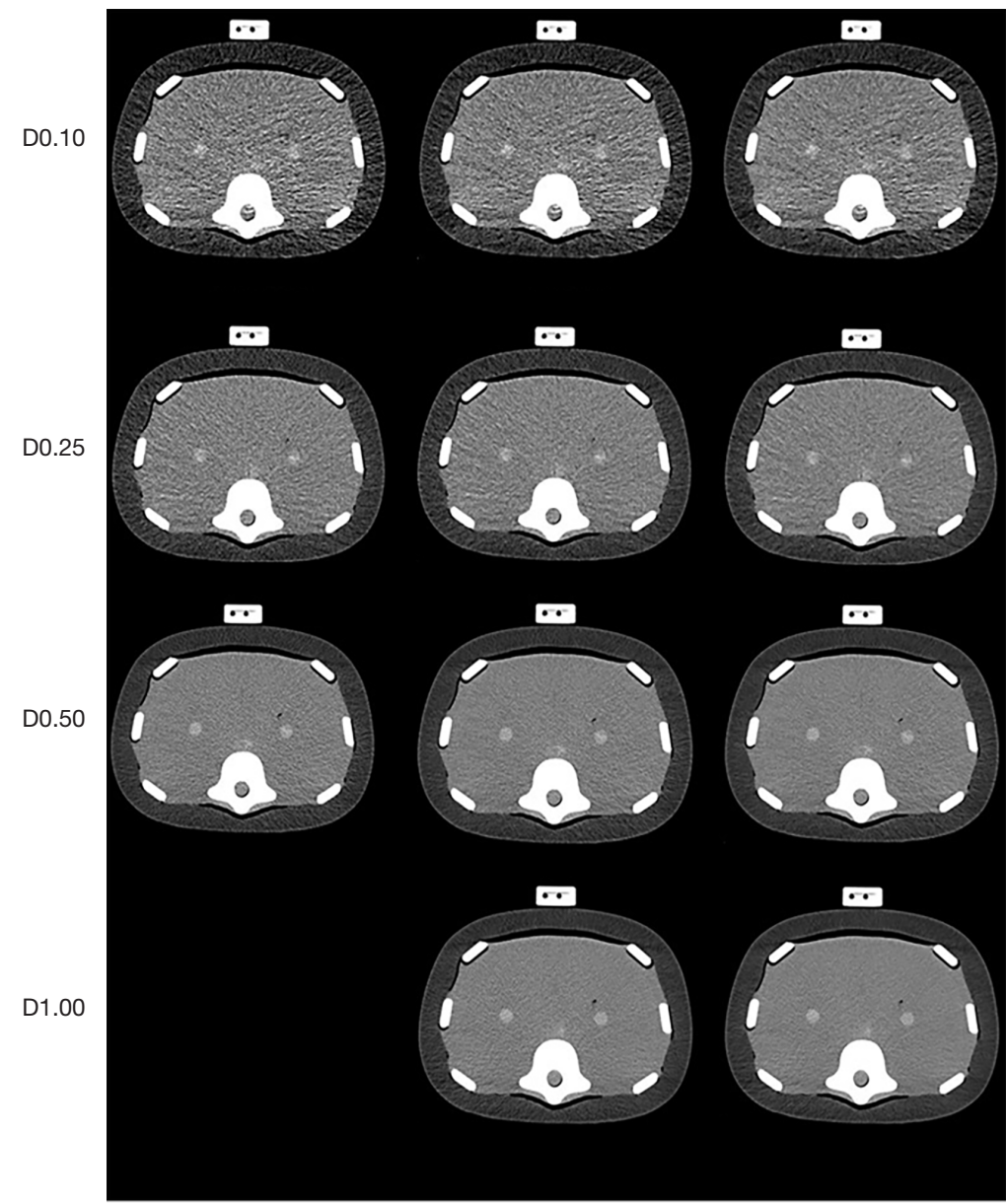

Figure S1 CT images at different ADMIRE levels and different dose levels, corresponding to Table S2. The two operators chose in consensus: D1.00: ADMIRE 2; D0.50: ADMIRE 3; D0.25: ADMIRE 4; D0.10: ADMIRE 5. CT, computed tomography; ADMIRE, advanced model-based iterative reconstruction. 


\section{IMACTIS ${ }^{\circledR}$ system (Figure S2)}

This system allows the interventional radiologist to explore the patient's anatomy in any plane and visualize the planned needle trajectory before its insertion in real-time.

The IMACTIS $^{\circledR}$ system is composed of a station with a touch screen and a proprietary electromagnetic locator. This locator is composed of a magnetic receiver, located inside a needle holder, providing good ergonomics. The magnetic transmitter, designed to be fixed to the phantom and detected in computed tomography (CT) images, allows an automatic registration between magnetic and CT coordinates. Once a CT-scan series (slice spacing of $2 \mathrm{~mm}$ maximum) is transferred to the navigation system, registration is automatically performed.

The touch screen is composed of two 2D reconstructed CT images extracted from the 3D CT volume that show the needle trajectory under two orthogonal views.

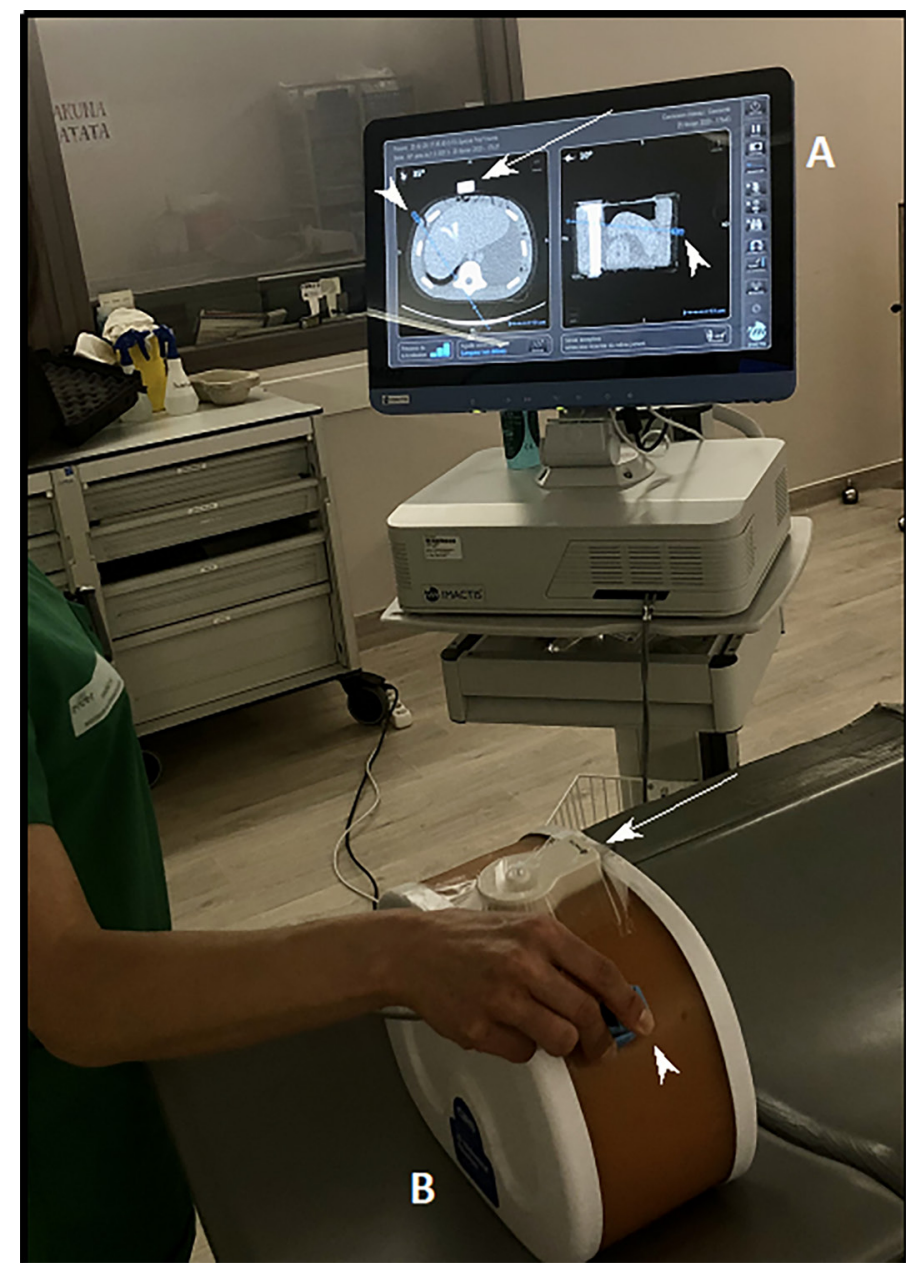

Figure S2 The Imactis ${ }^{\circledR}$ workstation, an example procedure. The Imactis ${ }^{\circledR}$ workstation (A) with one operator in action on phantom 2 (B). "A" represents the touch screen with two 2D-reconstructed CT images extracted from the 3D CT volume. The virtual needle path in two orthogonal views is virtually represented by a graduated blue line. The ergonomic needle holder handheld by the operator (blue holder with white arrow) is virtually projected onto the touch screen (white arrowhead). The magnetic transmitter is fixed to the phantom (white box with the white arrow) and detected in CT images (white arrow on the touch screen). CT, computed tomography. 